\title{
PRIME RINGS FOR WHICH THE SET OF NONZERO IDEALS IS A SPECIAL CLASS
}

\author{
B. J. GARDNER \\ (Received 13 February 1989; revised 31 August 1989) \\ Communicated by R. Lidl \\ Dedicated to the memory of Professor Kurt Mahler
}

\begin{abstract}
We show that the rings described in the title are precisely the indecomposable injectives for the category whose objects are the associative rings and whose morphisms are the ring homomorphisms with accessible images. These rings are more or less completely known. Those of cardinality greater than that of the continuum are subdirectly irreducible but there are some nontrivial principal ideal domains in the class.
\end{abstract}

1980 Mathematics subject classification (Amer. Math. Soc.) (1985 Revision): 16 A 21.

Throughout this note, all rings are associative but are not required to have an identity.

Since a class $\mathscr{H}$ of prime rings is special if and only if it is hereditary with respect to nonzero ideals and closed under essential extensions (see [3] for this characterization, which differs slightly from the original definition given by Andrunakievich [1]) it is clear that every nonempty intersection of special classes is special, and in particular every prime ring $A$ generates a special class (which, following [6] and [2], we shall call $\pi_{A}$ ). This $\pi_{A}$ is the intersection of all special classes containing $A$.

Ryabukhin [6] has obtained the following characterization of $\pi_{A}$ (see also [2, page 239]).

For a prime ring $A, \pi_{A}$ contains a prime ring $B$ if and only if $B$ has $a$ nonzero ideal which is isomorphic to an accessible subring of $A$.

(C) 1991 Australian Mathematical Society 0263-6115/91 \$A2.00+0.00 
Useful though it is, this description of $\pi_{A}$ is not overly explicit. For instance, one sees easily that if $A$ is simple, then $\pi_{A}$ consists of all subdirectly irreducible rings with hearts isomorphic to $A$ (though this can easily be deduced by other means) but this does not really tell us much about the "size" of $\pi_{A}$. On the other hand, for any prime ring $A$, if $0 \neq I \triangleleft A$, then $|A| \leq 2^{|I|}$ (see [6] or [2, page 240]) so we can say something about the size of members of $\pi_{A}$.

Our concern in this note is with those $\pi_{A}$ which are in a sense as small as possible: with those $A$ for which $\pi_{A}$ is (up to isomorphisms) the set of nonzero ideals of $A$. Since $\pi_{A}$ is closed under essential extensions and every prime ring has an essential extension (which is a prime ring) with identity, it is clear that $A$ must have an identity. What we seek, therefore, is a characterization of the prime rings $A$ with identity such that whenever $B$ is prime with a nonzero ideal isomorphic to an accessible subring of $A$, $B$ itself is isomorphic to an ideal of $A$. (There is a slightly better-looking condition obtained by the substitution of "accessible subring" for "ideal" in a couple of places, but this turns out to be equivalent to the one we have enunciated.) Now what we have is starting to look vaguely like an injectivetype concept. Our main result will make this much more precise, and the relevant injectivity is the following.

A ring $R$ is acc-injective [4] if it is an injective object in the category whose objects are rings (not necessarily with identity) and whose morphisms are ring homomorphisms with accessible images. The acc-injectives have been fairly completely described in [4] and [5]. In particular, they have identities.

THEOREM. The following conditions are equivalent for a prime ring $A$ :

(i) $\pi_{A}$ is (up to isomorphisms) the set of nonzero ideals of $A$;

(ii) $\pi_{A}$ is (up to isomorphisms) the set of nonzero accessible subrings of $A$;

(iii) $A$ is acc-injective.

Proof. (i) $\Rightarrow$ (ii). This is clear since in any case $\pi_{A}$ contains all nonzero accessible subrings of $A$.

(ii) $\Rightarrow$ (iii). Let $A$ be a prime ring satisfying (ii). As noted above, $A$ has an identity. If $0 \neq I \triangleleft A$ then by factoring out of the standard unital extension of $I$ an ideal maximal with respect to having zero intersection with $I$, we obtain a ring $\bar{I}$ with identity in which $I$ is an essential ideal. This $\bar{I}$ is prime and so, as $\bar{I} \in \pi_{A}, \bar{I}$ is (isomorphic to) an accessible subring of $A$. But $\bar{I}^{2}=\bar{I}$ so $\bar{I}$ is isomorphic to an ideal, hence a direct summand, of $A$, whence as $A$ is prime, $\bar{I} \cong A$. It follows that $A$ is generated, as a ring, by 
$I$ and 1 ; we denote this as follows:

$$
A=\langle I, 1\rangle \text { whenever } 0 \neq I \triangleleft A \text {. }
$$

If $0 \neq K \triangleleft I \triangleleft A$, then $A K+K A=\langle I, 1\rangle K+K\langle I, 1\rangle \subseteq K$. Thus

$$
\text { all accessible subrings of } A \text { are ideals. }
$$

Now consider two nonzero, isomorphic, ideals $I$ and $J$ of $A$. Since $I \cap J \neq 0$, we have

$$
\langle I, 1\rangle=\langle J, 1\rangle=\langle I \cap J, 1\rangle=A .
$$

Suppose $I \neq J$. Then we may assume that $J \nsubseteq I$. For every $j \in J$ (as $A=\langle I \cap J, 1\rangle)$ there exist $x \in I \cap J, k \in \mathbb{Z}$ such that $j=x+k \cdot 1$. Then $k \cdot 1=j-x \in J$, so as $J \neq I \cap J$, at least one such $k$ is nonzero. Clearly $\{n \in \mathbb{Z}: n \cdot 1 \in J\} \triangleleft \mathbb{Z}$, so taking $n_{0}$ as the positive generator for this ideal we see that (by (1)) every element of $A$ has a unique representation

$$
j+n \cdot 1, \quad j \in J, \quad n \in \mathbb{Z}, \quad 0 \leq n<n_{0} .
$$

Now $I$ and $J$ are isomorphic rings, so let $f: J \rightarrow I$ be a ring isomorphism. Then $f\left(n_{0} \cdot 1\right)=i_{0}$ for some $i_{0} \in I$, and then for every element $f(j)$ of $I$ we have

$$
i_{0} f(j)=f\left(n_{0} \cdot 1\right) f(j)=f\left(n_{0} j\right)=n_{0} f(j)=\left(n_{0} \cdot 1\right) f(j) .
$$

But then $\left(i_{0}-n_{0} \cdot 1\right) I=0$, so, $A$ being a prime ring, $i_{0}-n_{0} \cdot 1=0$. Hence $\{n \in \mathbb{Z}: n \cdot 1 \in I\}$ is an ideal of $\mathbb{Z}$ containing $n_{0}$. Let $m_{0}$ be the positive generator of this ideal. Then $m_{0} \leq n_{0}$ and every element of $A$ has a unique representation

$$
i+n \cdot 1, \quad i \in I, \quad n \in \mathbb{Z}, \quad 0 \leq n<m_{0} .
$$

But by interchanging the roles of $I$ and $J$ in the isomorphism argument used above, we can deduce that $n_{0} \leq m_{0}$. Thus we get

$$
A=\left\{i+n \cdot 1: i \in I, 0 \leq n<n_{0}\right\}=\left\{j+k \cdot 1: j \in J, 0 \leq k<n_{0}\right\},
$$

where $i$ and $n, j$ and $k$ are unique. The first part of the argument just given also shows that

$$
f\left(n_{0} \cdot 1\right)=n_{0} \cdot 1 \text {. }
$$

We extend our isomorphism $f$ to a map $\hat{f}: A \rightarrow A$ by the rule

$$
\hat{f}(j+n \cdot 1)=f(j)+n \cdot 1\left(j \in J, n \in \mathbb{Z}, \quad 0 \leq n<n_{0}\right) .
$$

By (3), $\hat{f}$ is well defined. Let $n, n^{\prime} \in \mathbb{Z}$ be such that $0 \leq n<n_{0}, 0 \leq n^{\prime}<$ $n_{0}$, and let $n+n^{\prime}=k n_{0}+r$, where $0 \leq r<n_{0}$. Then (for $j, j^{\prime} \in J$ ) we 
have

$$
\begin{aligned}
\hat{f}(j+n \cdot 1)+\hat{f}\left(j^{\prime}+n^{\prime} \cdot 1\right) & =f(j)+n \cdot 1+f\left(j^{\prime}\right)+n^{\prime} \cdot 1 \\
& =f(j)+f\left(j^{\prime}\right)+\left(n+n^{\prime}\right) \cdot 1 \\
& =f(j)+f\left(j^{\prime}\right)+k n_{0} \cdot 1+r \cdot 1 \\
& =f(j)+f\left(j^{\prime}\right)+f\left(k n_{0} \cdot 1\right)+r \cdot 1(\mathrm{by}(4)) \\
& =f\left(j+j^{\prime}+k n_{0} \cdot 1\right)+r \cdot 1 \\
& =\hat{f}\left(j+j^{\prime}+k n_{0} \cdot 1+r \cdot 1\right) \\
& =\hat{f}\left(j+j^{\prime}+\left(n+n^{\prime}\right) \cdot 1\right) \\
& =f\left(j+n \cdot 1+j^{\prime}+n^{\prime} \cdot 1\right)
\end{aligned}
$$

and similarly one shows that $\hat{f}$ preserves multiplication. Thus $\hat{f}$ is a homomorphism. From (3) and the fact that $f$ is an isomorphism, it is clear that $\hat{f}$ is in fact an isomorphism, so

$$
\begin{aligned}
& \text { every isomorphism between ideals of } A \text { extends to an auto- } \\
& \text { morphism of } A \text {. }
\end{aligned}
$$

Now let $R$ be a prime ring having a nonzero ideal $L$ isomorphic to an ideal $I$ of $A$, and let $g: L \rightarrow I$ be an isomorphism. Then $R$ is in $\pi_{A}$ so by assumption (that is, (ii)) and (2), there is an injective homomorphism $h: R \rightarrow A$ such that $h(R) \triangleleft A$ and then $h(L) \triangleleft h(R) \triangleleft A$ so $h(L) \triangleleft A$. But $h(L) \cong I$ via the correspondence

$$
x \mapsto g h^{-1}(x) .
$$

Hence by (5) there is an automorphism $F: A \rightarrow A$ such that $F(x)=g h^{-1}(x)$ for all $x \in h(L)$. Now $F h$ is an injective homomorphism and $F h(R) \triangleleft A$. Since for each $z \in L$ we have $F h(z)=g h^{-1} h(z)=g(z)$ we have the following commutative diagram

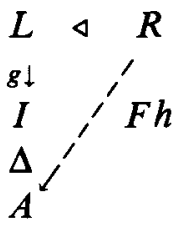

(with $F h(R) \triangleleft A$ ). This shows that $A$ satisfies a "special case" of accinjectivity. We now consider the general case.

Let $T$ be any ring, $0 \neq V \triangleleft T$ and let $G: V \rightarrow A$ be a homomorphism with $G(V)$ an accessible subring and therefore an ideal of $A$ (by (6)). Then $G(V)$ is a prime ring so $\operatorname{Ker}(G)$ is an ideal of $T$. Let us re-name $V / \operatorname{Ker}(G)$ and $T / \operatorname{Ker}(G)$ as $V_{1}, T_{1}$ respectively. Now $V_{1} \triangleleft T_{1}$. Let $M$ be an ideal of $T_{1}$ which has zero intersection with $V_{1}$ and is maximal for this. Then 
$T_{1} / M$ is an essential extension of $\left(V_{1}+M\right) / M \cong V_{1}$, so $T_{1} / M$ is a prime ring. Since $\left(V_{1}+M\right) / M \cong V_{1}=V / \operatorname{Ker}(G) \cong G(V) \triangleleft A$ there is an injective homomorphism $H: T_{1} / M \rightarrow A$ with $H\left(T_{1} / M\right) \triangleleft A$ such that $H$ extends the map of $\left(V_{1}+M\right) / M$ into $A$ (by the "special case" above). In other words we have a commutative diagram

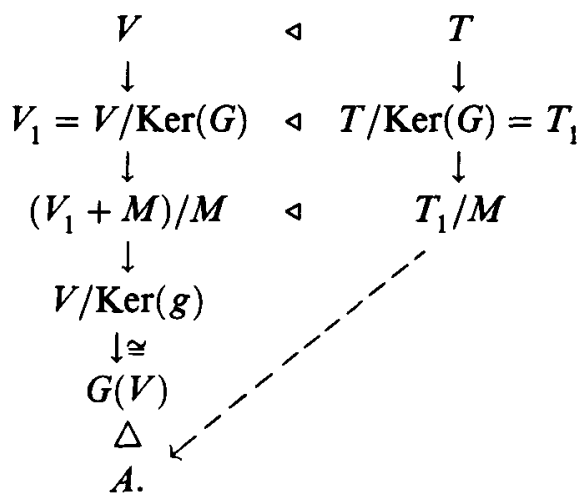

By induction we can replace the condition " $V \triangleleft T$ " with " $V$ is an accessible subring of $T "$. This proves that $A$ is acc-injective.

(iii) $\Rightarrow$ (i). Let $A$ be acc-injective, $B \in \pi_{A}$. Then $B$ has a nonzero ideal $I$ which is isomorphic to an accessible subring of $A$. Thus there is an injective homomorphism $g: I \rightarrow A$ with $g(I)$ an accessible subring of $A$. Then there is a homomorphism $h: B \rightarrow A$ with accessible image such that $h(i)=g(i)$ for all $i \in I$. This latter condition requires that $I \cap \operatorname{Ker}(h)=0$, whence, $B$ being a prime ring, $\operatorname{Ker}(h)=0$ and $B$ is isomorphic to an accessible subring of $A$ and hence, by (2), an ideal of $A$.

From [4] and [5] we know that the prime (or, equivalently, indecomposable) acc-injectives are

(i) the unital simple rings,

(ii) the standard characteristic $p$ unital extensions of certain simple rings of characteristic $p$ which do not admit algebra structures over fields other than $\mathbb{Z}_{p}$ and

(iii) rings (they are in fact principal ideal domains) all of whose proper homomorphic images are isomorphic to rings $\mathbb{Z}_{n}$ (various $n$ ).

A ring $A$ of type (iii) is embeddable in the ring of $p$-adic integers whenever $p A \neq A$ and thus $|A| \leq 2^{\aleph_{0}}$. Hence we have

COROLLARY. Every prime ring which satisfies the conditions of the Theorem and has greater cardinality than the continuum is subdirectly irreducible. 


\section{References}

[1] V. A. Andrunakievič, 'Radicals of associative rings I', Amer. Math. Soc. Transl. (2) 52 (1966), 95-128.

[2] V. A. Andrunakievich and Yu, M. Ryabukhin, Radicals of algebras and structure theory (in Russian), Nauka, Moscow, 1979.

[3] B. J. Gardner, 'Injectives for ring monomorphisms with accessible images', Comm. Algebra 10 (1982), 673-694.

[4] B. J. Gardner and P. N. Stewart, 'Injectives for ring monomorphisms with accessible images, II', Comm. Algebra 13 (1985), 133-145.

[5] G. A. P. Heyman and C. Roos, 'Essential extensions in radical theory', J. Austral. Math. Soc. (Series A) 23 (1977), 340-347.

[6] Yu. M. Ryabukhin, 'Supernilpotent and special radicals', (in Russian), Mat. Issled. 48 (1978), 80-93.

Department of Mathematics

University of Tasmania

G.P.O. Box 252C

Hobart Tasmania 7001

Australia 